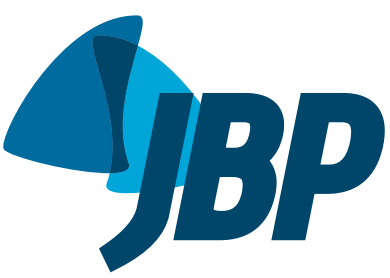

\title{
Thermography as a tool for monitoring the interface between the noninvasive ventilation mask and the skin
}

\author{
Bruno do Valle Pinheiro'
}

Noninvasive ventilation (NIV) is an important supportive measure for patients with acute respiratory failure. In patients with COPD exacerbation or acute cardiogenic pulmonary edema, for example, NIV is associated with reduced mortality and decreased need for intubation, its use therefore being recommended.(1) In patients with asthma or pneumonia, as well as in the prevention of weaning failure, NIV has been reported to improve clinical and functional parameters and can be used on the basis of clinical judgment. (2)

Although NIV plays an important role in the treatment of acute respiratory failure, NIV failure rates are relatively high, ranging from $5 \%$ to $40 \%$. ( $^{(3)}$ In addition, mortality rates tend to be higher in patients in whom attempts at NIV fail than in those who are intubated without attempt at NIV. ${ }^{(4)}$ Therefore, it is important to identify the risk factors for NIV failure.

Causes of NIV failure include interface-related issues such as air leaks. ${ }^{(3)}$ More often than not, the NIV interface is adjusted incorrectly in an attempt to reduce air leaks, thus resulting in excessive pressure on the skin and, consequently, pressure ulcers, particularly on the nasal dorsum, which is a poorly vascularized area with little tissue between the skin surface and underlying bone. ${ }^{(5)}$

In the current issue of the JBP, Pontes et al.(6) published the results of a clinical study in which they used a new technology known as skin thermometry or thermography (infrared imaging of the skin) in order to evaluate the effects of NIV on the skin where it was in contact with the interface (an oronasal mask). The two regions of interest were the nasal dorsum and the area of contact between the skin surface and the oronasal mask, the effects being compared between healthy individuals receiving continuous positive airway pressure (CPAP) or bilevel positive airway pressure (BiPAP). The participants $(n=20)$ were randomized to receive CPAP or BiPAP for $60 \mathrm{~min}$, the exclusion criteria being as follows: having a dermatologic disease, a neurological disease, diabetes, nutritional disorders, or dehydration; currently using creams or chemical substances on the face; being on corticosteroids, anti-inflammatory drugs, or antihistamines; having an axillary temperature outside the normal range; and having excessive sun exposure, which was defined as direct, unprotected sun exposure for more than $30 \mathrm{~min}$ before the experiment. CPAP was set at $10 \mathrm{cmH}_{2} \mathrm{O}$, whereas BiPAP was set to deliver an expiratory pressure of $5 \mathrm{cmH}_{2} \mathrm{O}$ and an inspiratory pressure of $20 \mathrm{cmH}_{2} \mathrm{O}$ (pressure support, $15 \mathrm{cmH}_{2} \mathrm{O}$ ). In both groups, the interface for NIV was an oronasal mask. A questionnaire was used in order to determine whether participants had experienced any adverse effects (skin lesions, pain, or other adverse effects). Thermographic imaging was used in order to determine skin temperature, which was subsequently analyzed for correlations with adverse events.

Adverse effects were found to be more intense in the individuals receiving BiPAP than in those receiving CPAP. This is probably due to the fact that pressure levels were higher in the BiPAP group than in the CPAP group, an inspiratory positive airway pressure of $20 \mathrm{cmH}_{2} \mathrm{O}$ being delivered to the individuals in the BiPAP group. In addition, all pressure levels were preset, no adjustments being made to improve patient comfort. In a study published in 2009, a group of authors demonstrated that an increased inspiratory pressure is associated with interface-related side effects, a finding that was confirmed in other studies. ${ }^{(7,8)}$

The most significant contribution of the study by Pontes et al. ${ }^{(6)}$ was their evaluating the effects of NIV on skin temperature where the skin was in contact with the interface, in an attempt to understand the impact of NIV modes and settings on the local skin microcirculation, as indirectly assessed by infrared thermographic imaging. One interesting finding was a reduction in skin temperature in the areas of contact between the face and the mask and between the nasal dorsum and the mask. The reduction was less pronounced in the area of contact between the nasal dorsum and the mask. However, the baseline temperature of the nasal dorsum skin was lower than was that of the facial skin. This finding was expected and suggests reduced blood flow, probably due to the pressure exerted by the mask. However, it is possible that the airflow provided by the ventilator contributed to the cooling of the skin. Nevertheless, the reduction in skin temperature was found to be less pronounced in the CPAP group than in the BiPAP group, a finding that suggests that the cooling was likely due to ischemia rather than the airflow provided by the ventilator. Given that the airflow provided by the ventilator tends to be higher for BiPAP than for CPAP, particularly at high pressures such as those used in the study by Pontes et al., ${ }^{(6)}$ its role in cooling the skin should have been more significant in the BiPAP group than in the CPAP group.

Another interesting finding was related to skin temperature after mask removal. In the area of contact between the face and the mask, skin temperature increased at 5 min after mask removal, continuing to rise over the course of the 30 -min follow-up period. This increase in local skin temperature was probably due to flows returning to appropriate perfusion levels or even to increased perfusion caused by reactive hyperemia. Although this is expected in situations such as this, 
the same was not true for the nasal dorsum, where skin temperature decreased immediately after mask removal and remained low over the course of the 30-min follow-up period. This finding suggests that skin perfusion was less efficient in the nasal dorsum region, particularly in the presence of pressure levels that can cause ischemia, and explains why pressure ulcers are more likely to occur in that area during NIV, constituting the most significant contribution of the study in my opinion.

According to the authors themselves, one of the limitations of the study was the small sample size, especially if we take into account that the response varied among individuals. Another limitation lies in the possibility that thermography was affected by the temperature of the airflow provided by the ventilator. If that is the case, the use of heated humidification and evaluation of the areas to which the airflow provided by the ventilator was delivered but on which the mask exerted no pressure might aid in clarifying this issue. Finally, the study findings have limited clinical applicability because NIV duration was not long enough to evaluate the studied complications and because the study included healthy individuals (although the effects of NIV on microcirculation might be even worse in diseased individuals). However, none of the aforementioned limitations diminish the relevance of the study findings, because of the pioneering use of a new noninvasive method for monitoring an important side effect of NIV.

\section{REFERENCES}

1. Barbas CS, Isola AM, Farias AM, Cavalcanti AB, Gama AM, Duarte $A C$, et al. Brazilian recommendations of mechanical ventilation 2013. Part I. Rev Bras Ter Intensiva. 2014; 26(2):89-121. https://doi. org/10.5935/0103-507X.20140017

2. Cabrini L, Landoni G, Oriani A, Plumari VP, Nobile L, Greco M, et al. Noninvasive ventilation and survival in acute care settings: a comprehensive systematic review and metaanalysis of randomized controlled trials. Crit Care Med. 2015; 43(4):880-8. https://doi org/10.1097/CCM.0000000000000819

3. Hess DR. Noninvasive ventilation for acute respiratory failure. Respir Care. 2013; 58(6):950-72. https://doi.org/10.4187/respcare.02319

4. Stefan MS, Nathanson BH, Higgins TL, Steingrub JS, Lagu T, Rothberg $M B$, et al. Comparative effectiveness of noninvasive and invasive ventilation in critically ill patients with acute exacerbation of chronic obstructive pulmonary disease. Crit Care Med. 2015;43(7):1386-94
https://doi.org/10.1097/CCM.0000000000000945

5. Nava S, Navalesi P, Gregoretti C. Interfaces and humidification for noninvasive mechanical ventilation. Respir Care. 2009;54(1):71-84.

6. Pontes SM, Melo LH, Maia NP, Nogueira AN, Vasconcelos TB, Pereira ED, et al. Influence that the ventilatory mode has on acute adverse effects and facial thermography after noninvasive ventilation. J Bras Pneumol. 2017;43(2):87-94

7. Holanda MA, Reis RC, Winkeler GF, Fortaleza SC, Lima JW Pereira ED. Influence of total face, facial and nasal masks on short-term adverse effects during noninvasive ventilation. J Bras Pneumol. 2009;35(2):164-73. https://doi.org/10.1590/S180637132009000200010

8. Hess DR. Patient-ventilator interaction during noninvasive ventilation Respir Care. 2011;56(2):153-65; discussion 165-7. https://doi. org/10.4187/respcare.01049 